\title{
Perfiles de pacientes asociados con intento de suicidio impulsivo que acudieron al hospital Homero Castanier Crespo de ciudad de Azogues en el periodo 2010-2012
}

\section{Profiles of patients associated with impulsive suicide attempt who attended the Homero Castanier Crespo hospital in the city of Azogues in the period 2010-2012}

Hugo Renán Ruiz López

Universidad Internacional del Ecuador, Ecuador

Adriana Priscila Villa Beltrán

Consultor Independiente, Ecuador

Diego Fernando Timbe Ávila

Consultor Independiente, Ecuador

Autor por correspondencia: huruizlo@uide.edu.ec

Fecha de recepción: 20 de Octubre de 2017 - Fecha de aceptación: 15 de Abril de 2018

\section{Resumen}

En el presente trabajo se estudia a 172 pacientes que acudieron al Hospital Homero Castanier Crespo de la ciudad de Azogues en el periodo 2010-2012 con diagnóstico de intento de suicidio, con el fin de describir, analizar y encontrar el perfil del paciente asociado con el intento de suicidio del tipo impulsivo, para ello se estudiaron 20 variables independientes y su asociación con la variable tipo de intento de suicidio, adicionalmente se realizó una segmentación bajo la metodología de árboles de decisión de los pacientes obteniendo segmentos asociados con el tipo de intento de suicidio .

Palabras Claves: intento de suicidio; perfiles de pacientes; prevalencia; suicidio impulsivo

\begin{abstract}
In the present work, we study 172 patients who attended the Homero Castanier Crespo Hospital in the city of Azogues in the period 2010-2012 with a diagnosis of attempted suicide, in order to describe, analyze and find the profile of the associated patient. with the suicide attempt of the impulsive type, for this 20 independent variables were studied and their association with the variable type of suicide attempt, additionally a segmentation was performed under the decision trees methodology of the patients obtaining segments associated with the type of suicide. suicide attempt.
\end{abstract}

Key words: attempt of suicide; patient profiles; prevalence; impulsive suicide 


\section{Introducción}

Se entiende por suicidio todo acto u omisión de este realizado voluntariamente con el propósito de dejar de existir, es decir, de quitarse la vida. Generalmente la persona que decide suicidarse tiende a estar pasando por un período de profundo sufrimiento vital en diferentes aspectos importantes de su vida, sin poder hacerle frente y estando en un estado de desesperación en el que la única salida que pueden ver es la muerte (Castillero Mimenza).

Cada suicidio es una tragedia que afecta a familias, comunidades y países y tiene efectos duraderos para los allegados del suicida. El suicidio se puede producir a cualquier edad, y en 2015 fue la segunda causa principal de defunción en el grupo etario de 15 a 29 años en todo el mundo (OMS, 2017).

El suicidio no solo se produce en los países de altos ingresos, sino que es un fenómeno global que afecta a todos los estratos de los países del mundo. De hecho, en 2015, más del 78\% de los suicidios de todo el mundo tuvieron lugar en países de ingresos bajos y medianos. (OMS, 2017).

El suicidio es una urgencia vital ubicada no sólo en un contexto biográfico de perdida de la salud de la persona, sino también de debilitamiento de sus redes afectivas y sociales (Echeburúa, 2015). A pesar de la eficacia de la actividad médica que ha contribuido que muchas enfermedades no incrementen la mortalidad, los daños intencionales como los homicidios y suicidios se ha incrementado hasta llegar a constituirse en unas de las principales causas de muerte en la población joven (Santiago Campbell, 1999).

Datos y Cifras (OMS, 2017)

- Cerca de 800.000 personas se suicidan cada año.

- Por cada suicidio, hay muchas más tentativas de suicidio cada año. Entre la población en general, un intento de suicidio no consumado es el factor individual de riesgo más importante.

- El suicidio es la segunda causa principal de defunción en el grupo etario de 15 a 29 años.

- El $78 \%$ de todos los suicidios se produce en países de ingresos bajos y medianos.

- La ingestión de plaguicidas, el ahorcamiento y las armas de fuego son algunos de los métodos más comunes de suicidio en todo el mundo.

\section{Clasificación del suicidio:}

Los autores determinan al suicidio en dos grandes grupos de clasificación como son: De acuerdo al origen y la forma:

- Por el Origen: se clasifican en Psicótico, neurótico psicodisplásico y fillosófico.

o Psicótico: el individuo se encuentra ajeno a su entorno y realidad, presenta ideas no lógicas y alucinantes.

o Neurótico: individuo con falta de intención real de lo que es el suicidio. 
o Psicodisplásico: se refiere a la persona psicótica con autoagresividad.

o Filosófico: Persona con un vacío en su existencia.

- Por la forma: se clasifican en Impulsivo, Obsesivo y Reflexivo.

- Impulsivo: Persona con actos inmediatos.

- Obsesivo: Ideas de autodestrucción constante.

○ Reflexivo: Frecuente en personas con depresión.

El Objetivo de este artículo es identificar el perfil de los pacientes ingresados al hospital Homero Castanier Crespo de Azogues en el periodo comprendido entre 2010-2012 con diagnóstico de intento de suicidio impulsivo.

\section{Suicidio impulsivo}

Aunque una persona tenga deseos de acabar con su vida, sin impulsividad y/o ansiedad no se suicidará ni llevará a cabo ningún tipo de acto violento, ya que no existe la decisión de la ejecución, el paso al acto, para que el paciente acabe con su vida, explica la doctora Belén Arranz, coordinadora de Psiquiatría del Instituto Europeo de Neurociencias (IDN) de Barcelona, España (informe21.com).

La impulsividad no es una enfermedad, es un síntoma integrante de gran parte de la patología psiquiátrica, que está asociado a trastornos como el suicidio, la depresión y la ansiedad, las adicciones, la bulimia, la esquizofrenia, el juego patológico o el trastorno por déficit de atención con hiperactividad entre otros. En este sentido, se puede afirmar que entre el $15 \%$ y el $25 \%$ de la población padece trastornos por impulsividad patológica (informe21.com).

De todas formas (y como casi todos hemos experimentado) la impulsividad no siempre es mala. A pesar de que tiende a considerarse como una característica negativa, puede desempeñar un importante papel en el comportamiento normal de las personas, puesto que la impulsividad moderada puede ser evaluada como un rasgo socialmente beneficioso y admirado (decisión, rapidez en las respuestas). La intensidad de la impulsividad es la que la convierte en patológica o disfuncional (con predisposición a reacciones rápidas, no planeadas, ante estímulos internos o externos, sin considerar las consecuencias negativas de esas acciones) (informe21.com).

Los resultados de un gran número de estudios no son consistentes con el punto de vista del continuo en la suicidalidad, indicando que en muchos casos el riesgo de intentar el suicido se desarrolla rápidamente con poca planificación previa o premeditación (Malo, 2015). La mayoría de los estudios (58\%) encuentra que los suicidios impulsivos constituyen más de la mitad de todos los intentos de suicidio. Los estudios de más calidad revisados dan una frecuencia de $51 \%$ de suicidios impulsivos y los de menos calidad del 65\%. Los estudios que revisan la planificación de los intentos concluyen que la planificación está ausente en dos tercios de los suicidios, lo que indica que la mayoría de los intentos muestran poca planificación previa (Malo, 2015). 
Otro hecho importante es que los estudios de suicido impulsivo señalan la importancia de algún tipo de factor estresante, principalmente factores interpersonales, en relación estrecha temporal con el acto. Muchas veces los suicidios impulsivos son una respuesta a estos conflictos interpersonales que han ocurrido en las horas previas al acto (Malo, 2015).

\section{Estudio del perfil asociado con diagnóstico de intento de suicidio impulsivo en los pacientes}

El presente artículo tiene como objetivo determinar el perfil asociado de los pacientes con intento de suicidio impulsivo, para ello se tomó como muestra 172 historias clínicas con diagnóstico de intento de suicidio del Hospital "Homero Castanier Crespo" de la ciudad de Azogues durante el periodo comprendido entre los años 2010 y 2012, en la cual incluía 20 variables del paciente.

Variables
\begin{tabular}{|l|l|l|l|}
\hline Edad & Residencia & Tentativas Previas & Mecanismo Utilizado \\
\hline Sexo & Ciudad & Alcoholismo y/o Drogadicción & Tipo de Mecanismo \\
\hline Instrucción & Estado Civil & Enfermedad Psiquiátrica & Tipo de Intento de Suicidio \\
\hline Ocupación & Etnia & Tipo de Alteración Psiquiátrica & Factor Desencadenante \\
\hline Actividad & Religión & Tratamiento (Psiquiatra) & Diagnóstico De Alta \\
\hline
\end{tabular}

Cuadro 1: Variables para el estudio

Se creó una variable binomial llamada "tipo de intento de suicidio", la cual contiene información si el intento de suicidio es "impulsivo o no impulsivo". Se aplicó la prueba Chi cuadrado para evaluar asociaciones con un nivel de significancia del 5\% de las variables independientes con la variable dependiente. De los 172 casos de intento de suicidio, 86 casos (50\%) fueron diagnosticados como intento de suicidio impulsivo, adicionalmente el $6 \%$ del total de intentos de fue consumado.

\section{Análisis univariado}

A continuación presentamos las características principales de los pacientes en las cuales podemos destacar que el $59 \%$ de los pacientes fue del sexo femenino, la edad de prevalencia fueron los menores de 20 años con 49\%, los niveles de instrucción secundaria y primaria registraron los mayores porcentajes con el $45 \%$ y $40 \%$ respectivamente, el $58 \%$ de los pacientes fueron de zona rural, la ocupación del paciente con mayor participación fueron empleados con el $41 \%$, seguido de los estudiantes con el $33 \%$ y el mecanismo más utilizado fueron los venenos y plaguicidas con el $77 \%$. 


\begin{tabular}{|c|c|c|}
\hline Característica & Frecuencia & Porcentaje \\
\hline \multicolumn{3}{|l|}{ Sexo } \\
\hline Femenino & 102 & $59 \%$ \\
\hline Masculino & 70 & $41 \%$ \\
\hline \multicolumn{3}{|l|}{ Edad } \\
\hline $12-20$ años & 84 & $49 \%$ \\
\hline 21-30 años & 46 & $27 \%$ \\
\hline 31-40 años & 16 & $9 \%$ \\
\hline $41-50$ & 8 & $5 \%$ \\
\hline$>50$ años & 18 & $10 \%$ \\
\hline \multicolumn{3}{|l|}{ Instrucción } \\
\hline Ninguna & 11 & $6 \%$ \\
\hline Primaria & 69 & $40 \%$ \\
\hline Secundaria & 77 & $45 \%$ \\
\hline Superior & 15 & $9 \%$ \\
\hline \multicolumn{3}{|l|}{ Residencia } \\
\hline Rural & 99 & $58 \%$ \\
\hline Urbana & 73 & $42 \%$ \\
\hline \multicolumn{3}{|l|}{ Ocupación } \\
\hline Ama de Casa & 38 & $22 \%$ \\
\hline Desempleado & 7 & $4 \%$ \\
\hline Empleado & 70 & $41 \%$ \\
\hline Estudiante & 57 & $33 \%$ \\
\hline \multicolumn{3}{|l|}{ Mecanismo Utilizado } \\
\hline Venenos y Plaguicidas & 132 & $77 \%$ \\
\hline Intoxicación Medicamentosa & 16 & $9 \%$ \\
\hline Otras Sustancias Tóxicas & 13 & $8 \%$ \\
\hline Resto & 11 & $6 \%$ \\
\hline
\end{tabular}

\section{Análisis bivariado}

En esta parte del trabajo se presenta la distribución conjunta de dos variables, se investiga la influencia de las variables independientes con respecto a la variable dependiente binomial "tipo de intento de suicidio"

\section{Edad del paciente}

La edad promedio en los pacientes con intento de suicidio impulsivo fue de 23 años, 8 años menos que los pacientes que fueron diagnosticados con otro tipo de intento de suicidio que registraron una edad promedio de 31 años. La edad mínima fue 12 años y la máxima 75 años.

La variable "edad" se asocia con la variable "tipo de intento de suicidio", de manera que la proporción de pacientes con intento de suicidio impulsivo es mayor en los pacientes menores a 23 años que en los pacientes mayores a esa edad, la prueba Chi Cuadrado fue de 9,834 con un $\underline{p}$

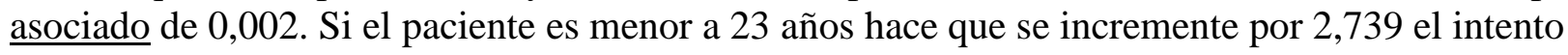
de suicidio "tipo impulsivo" 


\begin{tabular}{|c|c|c|c|}
\hline \multicolumn{4}{|c|}{ Estimación de riesgo } \\
\hline & \multirow[b]{2}{*}{ Valor } & \multicolumn{2}{|c|}{ Intervalo de confianza de $95 \%$} \\
\hline & & Inferior & Superior \\
\hline $\begin{array}{l}\text { Odds ratio para } \\
\text { Rango_Edad }(<=23 \text { I } \\
>23)\end{array}$ & 2,739 & 1,448 & 5,183 \\
\hline $\begin{array}{l}\text { Para cohorte } \\
\text { Tipo_Suicidio }=\text { Impulsivo }\end{array}$ & 1,705 & 1,183 & 2,458 \\
\hline $\begin{array}{l}\text { Para cohorte } \\
\text { Tipo_Suicidio = No } \\
\text { Impulsivo }\end{array}$ & .623 & .466 & 832 \\
\hline $\mathrm{N}$ de casos válidos & 172 & & \\
\hline
\end{tabular}

Cuadro 3: Odds Ratio Rango Edad vs Tipo Intento Suicidio

\section{Género del paciente}

De los 172 casos de intento de suicidio el 59\% fueron del género femenino y el $41 \%$ del género masculino. El 58\% de los intentos de suicidios en el género femenino fueron del tipo impulsivo, 19 puntos más que en el género masculino que registró el 39\% de intentos de suicidio de este tipo.

La variable género del paciente se asocia con la variable tipo de intento de suicidio, el valor de prueba Chi Cuadrado fue de 6.16 con un valor $\mathrm{p}$ asociado de 0.013 . Por otra parte la fuerza de asociación es de 2.185, que nos indica si ingresa un paciente con intento de suicidio del género femenino hace que se incremente por 2.184 el riesgo que sea del tipo impulsivo.

Estimación de riesgo

\begin{tabular}{|c|c|c|c|}
\hline & \multirow[b]{2}{*}{ Valor } & \multicolumn{2}{|c|}{ Intervalo de confianza de $95 \%$} \\
\hline & & Inferior & Superior \\
\hline $\begin{array}{l}\text { Odds ratio para Sexo } \\
\text { (Femenino / Masculino) }\end{array}$ & 2,185 & 1,174 & 4,068 \\
\hline $\begin{array}{l}\text { Para cohorte Intento } 2= \\
\text { Impulsivo }\end{array}$ & 1,500 & 1,069 & 2,105 \\
\hline $\begin{array}{l}\text { Para cohorte Intento } 2= \\
\text { No Impulsivo }\end{array}$ &, 686 & .512 &, 920 \\
\hline $\mathrm{N}$ de casos válidos & 172 & & \\
\hline
\end{tabular}

Cuadro 4: Odds Ratio Sexo vs Tipo Intento Suicidio

\section{Residencia del paciente}

De los 172 casos de intento de suicidio el $58 \%$ fueron pacientes del sector rural y el $42 \%$ del sector urbano. El 56\% de los intentos de suicidios en el sector urbano fueron del tipo impulsivo, 11 puntos más que en el sector rural que registró el $45 \%$ de intentos de suicidio de este tipo. 


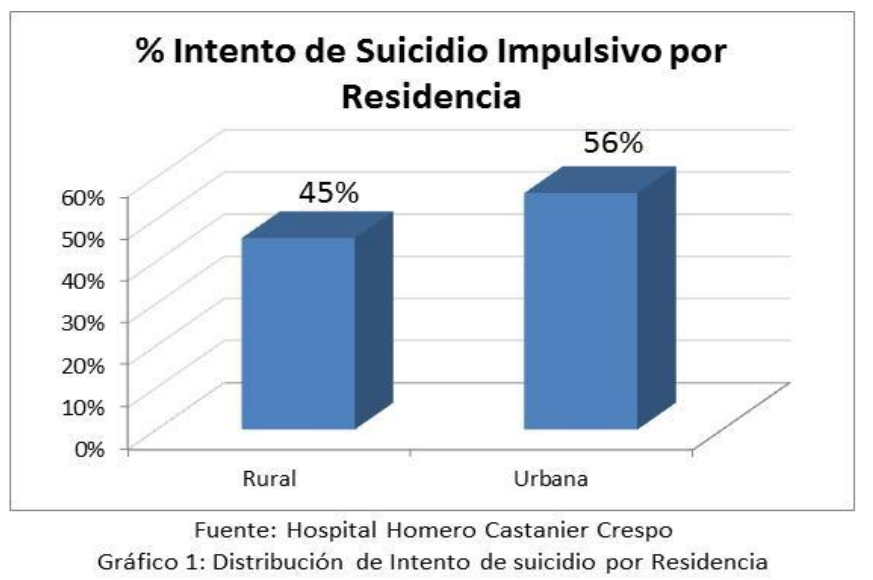

Adicionalmente se pudo obtener mediante una tabla de contingencia que los pacientes mayores a 22 años y del sector rural tienen 3,64 veces más posibilidades que el intento de suicidio sea del tipo no impulsivo que aquellos pacientes mayores de 22 años pero del sector urbano. El valor de la prueba Chi cuadrado fue de 6,301 con un valor p asociado de 0,012.

\section{Ocupación del paciente}

De los 172 casos de intento de suicidio 38 casos fueron amas de casa, 57 casos estudiantes, 70 casos empleados y 7 casos desempleados. Las ocupaciones de amas de casa y estudiantes registraron mayor porcentaje de intento de suicidio impulsivo con el $66 \%$ y $61 \%$ respectivamente, mientras los pacientes empleados y desempleados el menor porcentaje con el $36 \%$ y $14 \%$ cada uno.

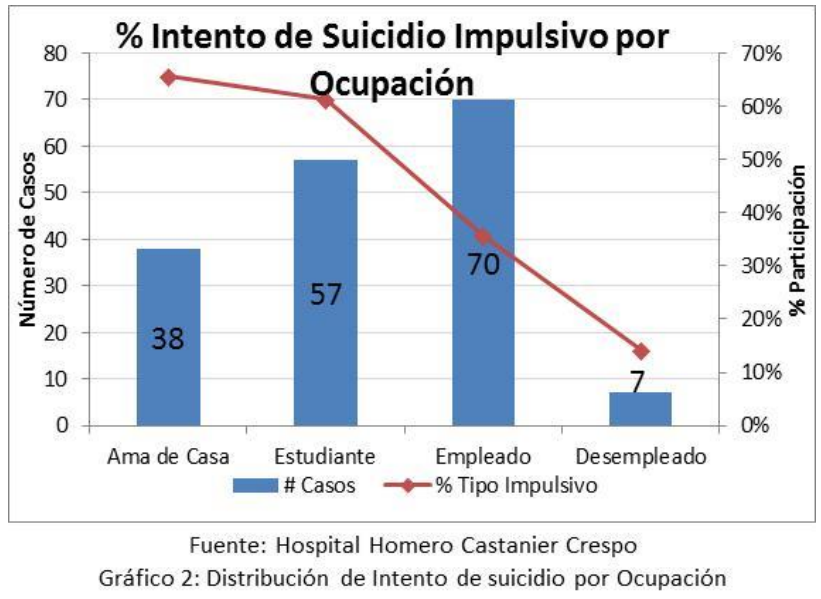

La variable ocupación del paciente se asocia con la variable tipo de intento de suicidio, el valor de prueba Chi Cuadrado fue de 16,040 con un valor p asociado de 0,001. Por otra parte la fuerza de asociación es de 3.363, que nos indica si ingresa un paciente con intento de suicidio es ama de casa o estudiante hace que se multiplique por 3.363 el riesgo que sea del tipo impulsivo.

\section{Perfil del paciente asociado con intento de suicidio impulsivo}


Mediante la metodología de árboles de decisión se obtuvieron los siguientes perfiles de pacientes con mayor prevalencia en el intento de suicidio del tipo impulsivo. A continuación presentamos los perfiles de los principales segmentos.

Perfilamos los pacientes en cinco segmentos, los dos primeros segmentos tienen un porcentaje de pacientes con diagnóstico de intento de suicidio impulsivo mayor al $60 \%$, estos pacientes tienen la característica que son menores a 22 años y profesión amas de casa o estudiantes, adicionalmente estos segmentos tienen una participación del $40 \%$ sobre el total de pacientes estudiados.

El segmento cinco registra el menor porcentaje de pacientes con diagnóstico de intento de suicidio impulsivo con el $24 \%$, estos pacientes tienen la característica de residir en zona rural y mayores a 22 años, la participación de este segmento es del $28 \%$ sobre el total de pacientes estudiados.

\begin{tabular}{|c|l|r|r|r|}
\hline Segmento & \multicolumn{1}{|c|}{ Perfil del Paciente } & \% Impulsivo & \# Pacientes & $\%$ Participación \\
\hline 1 & Edad <16 años, Ama de Casa o Estudiante & $76 \%$ & 33 & $19 \%$ \\
2 & Edad entre 16y 22 años, Ama de Casa o Estudiante & $61 \%$ & 36 & $21 \%$ \\
3 & Edad > 22 años, residencia urbana & $54 \%$ & 24 & $14 \%$ \\
4 & Edad entre 16y 22 años, diferente de Ama de Casa o Estudiante & $47 \%$ & 30 & $17 \%$ \\
5 & Edad > 22 años, residencia rural & $\mathbf{2 4 \%}$ & 49 & $\mathbf{2 8 \%}$ \\
\hline \multicolumn{2}{|c|}{ Total } & $\mathbf{5 0 \%}$ & $\mathbf{1 7 2}$ & $\mathbf{1 0 0 \%}$ \\
\hline
\end{tabular}

Cuadro 5: Segmentos de Pacientes de acuerdo al perfil asociado con intento de suicidio impulsivo

Para medir el ajuste del modelo matemático utilizamos el estadístico de prueba de Kolmogorov-Smirnov (coeficiente K-S), que mide la distancia máxima entre la función de distribución acumulada de pacientes con diagnóstico del tipo impulsivo y del tipo no impulsivo, entre mayor es la distancia o el K-S mejor es el modelo. Para ello contrastamos la siguiente prueba de hipótesis:

- $H_{0}$ : La distribución acumulada de los pacientes con diagnóstico del tipo impulsivo es equivalente a los del tipo no impulsivo.

- $H_{1}$ : No es verdad la hipótesis nula.

El K-S del modelo alcanza una máxima distancia de 0.314, mientras el valor del estadístico de prueba es 0.000 , el cual nos indica que existen diferencias significativas entre las distribuciones acumuladas de los tipos de suicidio, lo que equivale a rechazar la hipótesis nula que la distribución acumulada de pacientes con diagnóstico del tipo impulsivo y no impulsivo son equivalentes. 
Estadisticos de prueba ${ }^{a}$

\begin{tabular}{|ll|r|}
\hline & & Segmento \\
\hline Máximas diferencias & Absoluta &, 314 \\
extremas & Positivo &, 000 \\
& Negativo &,- 314 \\
Z de Kolmogorov-Smirnov & & 2,059 \\
Sig. asintótica (bilateral) & &, 000 \\
\hline
\end{tabular}

a. Variable de agrupación: Tipo_Suicidio

Cuadro 6: Resultado Prueba K-S

El Siguiente gráfico nos muestra que la distancia máxima o K-S en las distribuciones acumuladas, esta distancia máxima se registra en el segmento 3.

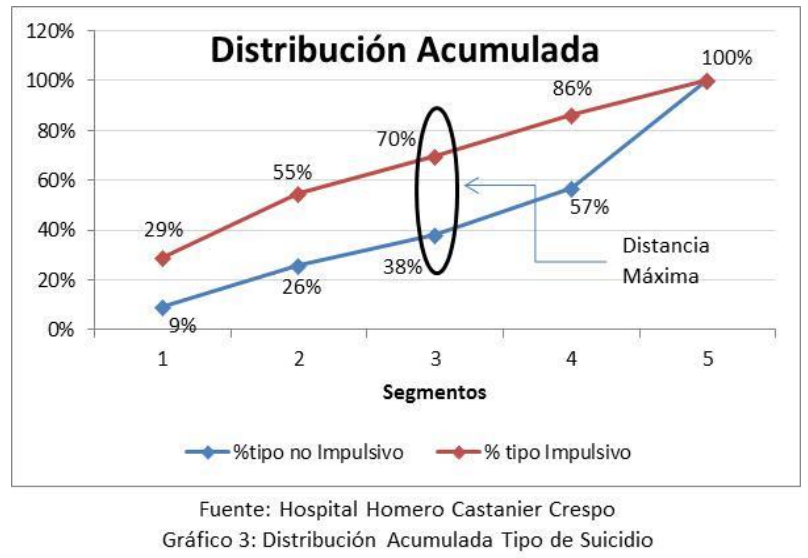

\section{Conclusiones}

Generalmente el intento de suicidio es más prevalente en mujeres que en los hombres, en el estudio presentando se confirma esto ya que de los 172 casos estudiados el 59\% (102 casos) fueron del género femenino y el $41 \%$ ( 70 casos) del género masculino.

Del total de pacientes ingresados por intento de suicidio el $77 \%$ fue por ingesta de sustancia toxica, es decir por envenenamiento.

El 49\% de los intentos de suicidio se dio en pacientes menores de 20 años, es decir que los adolescentes constituyeron el segmento de mayor riesgo.

El 50\% (86 casos) fueron diagnosticado como intento de suicidio impulsivo.

Si el paciente con intento de suicidio es menor a 23 años, es 2.7 veces más probable que el intento de suicidio sea del tipo impulsivo.

Si ingresa un paciente con intento de suicidio del género femenino, es 2.1 veces más probable que el riesgo sea del tipo impulsivo. 
El intento de suicidio del tipo impulsivo es más prevalente en el segmento de los menores a 16 años y de profesión ama de casa o estudiantes con el 76\% (25 de 33 casos).

El intento de suicidio del tipo impulsivo donde menos prevalece es los pacientes mayores a 22 años y residentes en zona rural con el $24 \%$ (12 de 49 casos).

\section{Bibliografía}

Aguayo Canela, M., \& Lora Monge, E. (2012). Como hacer una Regresión Logística . Fabis.

Carlos Gómez, N. R. (2002). Factor asociado al intento de suicidio en la población colombiana. Revista Colombiana de Psiquiatría, 271-286.

Castillero Mimenza, O. (s.f.). Los 26 tipos de suicidio. Obtenido de Los 26 tipos de suicidio: https://psicologiaymente.net/clinica/tipos-de-suicidio

Daniel Silva, B. V. (2013). Conducta Suicida y Trastornos Psiquiátricos en Chile, un estudio poblacional. Rev Med Chile, 1275-1282.

Echeburúa, E. (2015). Las múltiples caras del suicidio en la clínica psicológica. TERAPIA PSICOLÓGICA.

Gorricho, A. E. (2010). Concepto Y Clasificación De La Conducta Suicida. Alfonso Echávarri Gorricho.

Hernández, P. J. (s.f.). El Proceso Suicida.

informe21.com. (s.f.). Obtenido de https://informe21.com/impulsividad/existen-datos-cientificosdemuestran-impulsividad-suicidio-van-mano

Jorge Téllez-Vargas, M. (s.f.). Impulsividad y suicidio.

Malo, P. (2015). Evolución y Neurociencias. Obtenido de https://evolucionyneurociencias.blogspot.com/2015/04/el-suicidio-impulsivo.html

OMS. (2017). Suicidio. Obtenido de Organización Mundial de la Salud: http://www.who.int/mediacentre/factsheets/fs398/es/

Salazar, J. A. (2012). Aspectos Psicosociales del Comportamiento Suicida en Adolecentes. Psicología Iztacala, 688-721.

Santiago Campbell, C. d. (1999). Comportamiento de los intentos de suicidios letales y no letales. Acta Médica Colombiana Vol. $24 N^{\circ} 4$. 